\title{
A COMPARISON STUDY BETWEEN COLLINEARITY CONDITION, COPLANARITY CONDITION, AND DIRECT LINEAR TRANSFORMATION (DLT) METHOD FOR CAMERA EXTERIOR ORIENTATION PARAMETERS DETERMINATION
}

\author{
Khalid L. A. EL-ASHMAWY \\ Department of Civil Engineering, Al-Matria Faculty of Engineering, Helwan University \\ Matria, 11718 Cairo, EGYPT \\ Department of Civil Engineering, College of Engineering and Islamic Architecture, \\ Umm Al-Qura University, Makkah, Saudi Arabia \\ E-mail:khalid85_2002@yahoo.com
}

Received 27 December 2014; accepted 16 March 2015

\begin{abstract}
The present work emphasizes on using collinearity condition, coplanarity condition and DLT method for determining the camera exterior orientation parameters. The derivation of the mathematical formulation based on each suggested methods is explained. The comparison of the results of the methods was performed based on accuracy aspects using mathematical and actual photogrammetric data. The used data shows that the suggested methods are suitable for camera exterior orientation parameters determination for a block of photographs of any size. The results of this investigation prove that the accuracy of using coplanarity equations is slightly better than using collinearity equations or DLT method. Although the results of the DLT method are less accurate than those of using collinearity or coplanarity equation, DLT method is essential when the necessary information for the collinearity or coplanarity model is not available. This paper shows the necessity for the mathematical photogrammetric data for testing the photogrammetric studies. Keywords: collinearity condition; coplanarity condition; DLT method; camera exterior orientation; mathematical photogrammetric data.
\end{abstract}

\section{Introduction}

With regard to a single photograph, its exterior orientation (with respect to the object space) consists of two easily separable sets of parameters. One, involves the angles of rotation $(\omega, \varphi, \kappa)$ of the camera axis at the time of exposure and the other, the positional data of the exposure station in terms of the three-dimensional coordinates of the perspective centre $\left(X_{0}, Y_{0}, Z_{0}\right)$.

In topographic applications of photogrammetry, photogrammetrists think most naturally in terms of models produced by pairs of photographs. However, undoubtedly the most flexible approach to block formation and adjustment and to photogrammetry in general is through the use of the bundles of rays produced by individual photographs. In close range photogrammetry, where multi-station and convergent configurations are possible, the bundle approach can be seen in its most powerful form. The bundle approach is still the most flexible available approach and one that should give the best results.

The adjustment of the bundles in a block of photographs involves the rotation and translation of each bundle in space into such a position that all rays passing through the photographic positions of each control point will intersect at its correct object space position. Furthermore, all rays representing other points, such as a pass point, must intersect at their respective position in the object space.

The method of bundle adjustment makes use of the collinearity condition (El-Ashmawy, Azmi 2003; El-Ashmawy 1999), coplanarity condition (El-Ashmawy, Azmi 2003), or direct linear transformation method (El-Ashmawy 2006). The desired parameters (which include object space coordinates of new points which their object space coordinates are unknown, and camera exterior orientation parameters) are adjusted 
as a result of one simultaneous least squares solution of all the photographs by an iterative method. The iterations are necessary because of the fact that the associated condition equations are non-linear. The results of the bundle adjustment of the block of photographs are camera exterior orientation parameters of each photograph and a listing of the object space coordinates of the measured new points as well as their statistical precision.

Instead of getting the object space coordinates of new points and camera exterior orientation parameters in one simultaneous least squares solution of all photographs, the solution can be divided into two separate steps as following:

- Bundle adjustment for the determination of the camera exterior orientation parameters and object space coordinates of very limited number of points such as the six Gruber points (Wolf et al. 2014); and

- Space intersection for the determination of object space coordinates of unlimited number of points of interest such as pass and/or tie points, and planimetric and topographic points.

The advantage of this solution is the computation of the object space coordinates of points of interest in a separate step because:

- Their very presence could weight the solution according to their locations;

- Their effect on the accuracy of the computed control, pass and/or tie points, and camera exterior orientation parameters;

- The incorporation of such these points into block bundle adjustment software would unduly complicate.

Several methods can be applied to determine the parameters of the camera exterior orientation utilizing analytical photogrammetry. Three basic conditions are widely used to compute the exterior orientation parameters. These conditions are known as collinearity, coplanarity (Ghosh 2005) and coangularity (Wolf et al. 2014; Elnima 2015) conditions. All solutions pertaining the above mentioned conditions use point coordinates as input data (Grussenmeyer, Khalil 2002).

GPS techniques can be also used for determining the exterior orientation parameters, but only for aerial photogrammetry. Using GPS allows direct transformation of points into the mapping coordinate system. The main advantage of this method is the limitation of the iterative computation traditionally used to determine exterior orientation parameters. As a result, initial values of exterior orientation parameters are not needed and the number of control points needed to compute these values is considerably reduced.

The present research aims to derive mathematical formulations, investigate and compare the accuracies of three solutions for camera exterior orientation determination which are based on collinearity condition, coplanarity condition, and direct linear transformation (DLT) method.

\section{Determination of camera exterior orientation parameters using the collinearity condition}

Simultaneous adjustment utilizes the well known collinearity condition to establish two equations for each measured image point, and provides a unique solution for the system of observation equations by the least squares method.

The condition of collinearity is that an object point $(\mathrm{P})$, its image point $(\mathrm{p})$ and the perspective centre $(\mathrm{O})$, must lie along the same line. Mathematically, this condition is expressed as (Ghosh 2005; Elnima 2015):

$$
\left.\begin{array}{l}
x_{p}=-f \frac{\left(X_{P}-X_{O}\right) m_{11}+\left(Y_{P}-Y_{O}\right) m_{12}+\left(Z_{P}-Z_{O}\right) m_{13}}{\left(X_{P}-X_{O}\right) m_{31}+\left(Y_{P}-Y_{O}\right) m_{32}+\left(Z_{P}-Z_{O}\right) m_{33}} \\
y_{p}=-f \frac{\left(X_{P}-X_{O}\right) m_{21}+\left(Y_{P}-Y_{O}\right) m_{22}+\left(Z_{P}-Z_{O}\right) m_{23}}{\left(X_{P}-X_{O}\right) m_{31}+\left(Y_{P}-Y_{O}\right) m_{32}+\left(Z_{P}-Z_{O}\right) m_{33}}
\end{array}\right\},
$$

where $x_{p}, y_{p}$ are the corrected photo coordinates (ElAshmawy 1999), $X_{P}, Y_{P}, Z_{P}$ are the object space coordinates of point $\mathrm{P}, X_{O}, Y_{O}, Z_{O}$ are the object space coordinates of the perspective centre $O, f$ is the calibrated focal length of the camera, and $m_{i j}(i=1,2,3 ; j=$ $1,2,3)$ are the elements of the orientation matrix $(M)$ of the photograph.

The linearized form of Equation (1), for least squares method solution, can be given by (Wolf $e t$ al. 2014):

where:

$$
V+B \cdot \Delta=\varepsilon,
$$

$\Delta$ is the correction vector to the current values set for the unknowns (camera interior orientation parameters, camera exterior orientation parameters of each photo and object space coordinates of points) in the iterative solution;

$B$ is the matrix of the partial derivatives of Equation (1) with respect to the unknowns;

$V$ is the residual vector, i.e., the correction vector to the observations; and

$\varepsilon$ is the discrepancy vector. 
Constraints are suggested to consider supplemental observation equations (El-Ashmawy 2006; El-Ashmawy, Azmi 2003; El-Ashmawy 1999; Ghosh 2005) arising from $a$ priori knowledge regarding the object space coordinates of the control points in Equation (2). Such supplemental equations can be written as follows:

$$
V^{c}-\Delta^{c}=\varepsilon^{c},
$$

where:

$\Delta^{c}$ is the vector of observational corrections to the object space coordinates of the control points; and $\varepsilon^{c}$ is the discrepancy vector, between observed values and current (in iterative solution) values of the object space coordinates of the control points.

Observation equations can be obtained by merging Equations (2) and (3) as:

$$
\left.\begin{array}{c}
V+B \cdot \Delta=\varepsilon \\
V^{c}-\Delta^{c}=\varepsilon^{c}
\end{array}\right\},
$$

where the matrices are as defined earlier.

The fundamental requirements in bundle adjustment are the estimates of the camera interior and exterior orientations parameters. Furthermore, depending on the specific approach taken, the estimates for object space coordinates of all pass and/or tie points may also be needed. Thus, a bundle procedure should include a feasible method of obtaining the necessary estimated (approximate) values initially. The importance of initial values being close to most probable values of unknowns needs no emphasis. Not only it reduces the number of iterations but ensures fast and accurate results.

More details for the determination of the initial values of camera exterior orientation parameters and the object space coordinates of pass and/or tie points can be found in El-Ashmawy (1999).

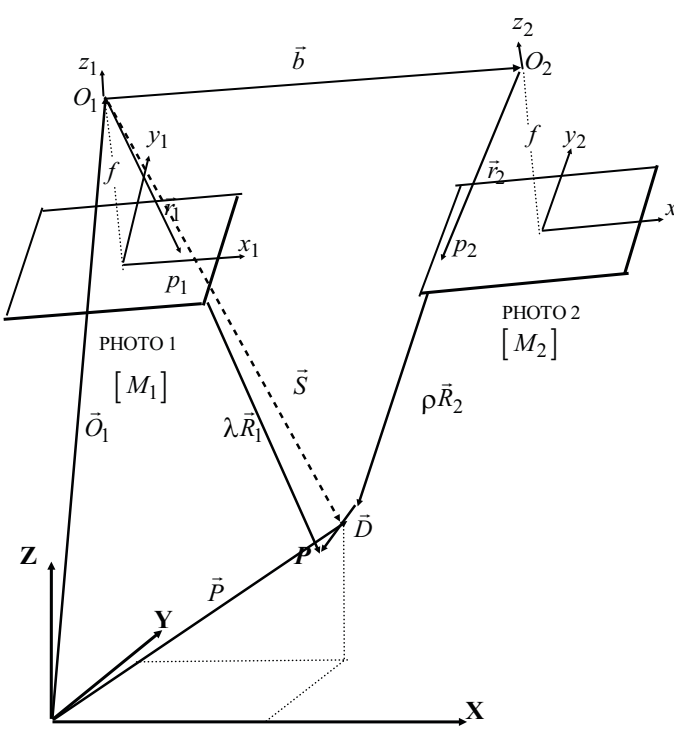

Fig. 1. Coplanarity Condition and Parallax

\section{Determination of camera exterior orientation parameters using the coplanarity condition}

The coplanarity condition (Fig. 1) implies that the two prespective centres, any object point and the corresponding image points on the two photographs of the stereo-pair, must all lie in a common plane. The coplanarity condition can be expressed as (El-Ashmawy 2006; Li, Zhao 2012; Wolf et al. 2014):

$$
F_{i}=\left|\begin{array}{ccc}
b_{X} & b_{Y} & b_{Z} \\
X_{1} & Y_{1} & Z_{1} \\
X_{2} & Y_{2} & Z_{2}
\end{array}\right|=0,
$$

where $b_{X}, b_{Y}, b_{Z}$ are the components of the air base vector $\vec{b}$, and $X_{1}, Y_{1}, Z_{1}$ and $X_{2}, Y_{2}, Z_{2}$ are the components of the vectors $\vec{R}_{1}$ (from $O_{1}$ to $P$ ) and $\vec{R}_{2}$ (from $\mathrm{O}_{2}$ to $P$ ) respectively.

The mathematical model consists of four scalar equations as follows:

$$
\left.\begin{array}{l}
X_{P}-\left(X_{O_{1}}+0.5\left(b_{X}+\lambda \cdot X_{1}+\rho \cdot X_{2}\right)\right)=0.0 \\
Y_{P}-\left(Y_{O_{1}}+0.5\left(b_{Y}+\lambda \cdot Y_{1}+\rho \cdot Y_{2}\right)\right)=0.0 \\
Z_{P}-\left(Z_{O_{1}}+0.5\left(b_{Z}+\lambda \cdot Z_{1}+\rho \cdot Z_{2}\right)\right)=0.0 \\
D_{Y}=\lambda \cdot X_{1}-\rho \cdot X_{2}-b_{Y}=0.0
\end{array}\right\},
$$

where $\left(X_{O_{1}}, Y_{O_{1}}, Z_{O_{1}}\right)$ are the object space coordinates of the first exposure station, and $\lambda$ and $\rho$ are scale factors of the corresponding location vectors $\vec{r}_{1}$ and $\vec{r}_{2}$ within the camera space.

The linearized form of Equation (6) with suggesting additional constraints to consider supplemental observation equations, for least squares method solution, can be given by:

where:

$$
\left.\begin{array}{l}
A \cdot V+B \cdot \Delta=\varepsilon \\
V^{c}-\Delta^{c}=\varepsilon^{c}
\end{array}\right\},
$$

$\Delta$ is the correction vector to the current values set for the unknowns (camera interior orientation parameters, camera exterior orientation parameters of the left and right photos and object space coordinates of points) in the iterative solution;

$A$ is the matrix of the partial derivatives of Equation (6) with respect to the observations (corrected photo coordinates on the left and right photos of the same object point);

$B$ is the matrix of the partial derivatives of Equation (6) with respect to the unknowns;

$V$ is the residual vector, i.e. the correction vector to the observations; and

$\varepsilon$ is the discrepancy vector.

$\Delta^{c}$ and $\varepsilon^{c}$ are as explained earlier. 
For starting the least squares iterative solution, the computation of the initial values of unknowns is essential and explained in (El-Ashmawy, Azmi 2003).

\section{Determination of camera exterior orientation parameters using the DLT method}

The Direct Linear Transformation (DLT) method was introduced to the photogrammetric community by (Abdel-Aziz, Karara 1971; Pannao, Pintavirooj 2012). DLT method models the transformation between the comparator or image pixel coordinate system and the object coordinate system as a linear function. DLT can be derived from the standard collinearity equations. The basic equations of DLT are expressed as follows (Abdel-Aziz, Karara 1971):

$$
\left.\begin{array}{l}
x=\frac{L_{1} X+L_{2} Y+L_{3} Z+L_{4}}{L_{9} X+L_{10} Y+L_{11} Z+1} \\
y=\frac{L_{5} X+L_{6} Y+L_{7} Z+L_{8}}{L_{9} X+L_{10} Y+L_{11} Z+1}
\end{array}\right\},
$$

where $x, y$ are the image coordinates, $L_{1}, \ldots, L_{11}$ are the transformation coefficients and $X, Y, Z$ are the object space coordinates of point.

The basic DLT equations (Equation (8)) actually contain 10 independent unknown parameters (Abdel-Aziz, Karara 1971). In other word one of the DLT parameters must be redundant and it is needed to add a non-linear constraint to the system to solve this problem. In this case, the method is called modified direct linear transformation (MDLT) method. The MDLT equations can be written as follows (El-Ashmawy 2006):

$$
\begin{aligned}
& x=\left(L_{4} L_{5} L_{10}^{2}+L_{4} L_{5} L_{11}^{2}-X L_{2} L_{6} L_{9}^{2}-X L_{2} L_{6} L_{11}^{2}-X L_{3} L_{7} L_{9}^{2}-\right. \\
& X L_{3} L_{7} L_{10}^{2}+X L_{2} L_{10} L_{5} L_{9}+X L_{2} L_{10} L_{7} L_{11}+X L_{3} L_{11} L_{5} L_{9}+ \\
& X L_{3} L_{11} L_{6} L_{10}+Y L_{2} L_{5} L_{10}^{2}+Y L_{2} L_{5} L_{11}^{2}-Y L_{2} L_{9} L_{6} L_{10}- \\
& Y L_{2} L_{9} L_{7} L_{11}+Z L_{3} L_{5} L_{10}^{2}+Z L_{3} L_{5} L_{11}^{2}-Z L_{3} L_{9} L_{6} L_{10}- \\
& \left.Z_{L_{3}} L_{9} L_{7} L_{11}-L_{4} L_{9} L_{6} L_{10}-L_{4} L_{9} L_{7} L_{11}\right) /(a+b)+ \\
& \xi\left(L_{12} r^{2}+L_{13} r^{4}+L_{14} r^{6}\right)+L_{15}\left(r^{2}+2 \xi^{2}\right)+L_{16} \xi \eta
\end{aligned}
$$$$
y=\frac{L_{5} X+L_{6} Y+L_{7} Z+L_{8}}{L_{9} X+L_{10} Y+L_{11} Z+1}+\eta\left(L_{12} r^{2}+L_{13} r^{4}+L_{14} r^{6}\right)+
$$$$
L_{15} \eta \xi+L_{16}\left(r^{2}+2 \eta^{2}\right) \text {, }
$$

where:

$$
\begin{aligned}
& a=\left(L_{5} L_{11}^{2}+L_{5} L_{10}^{2}+X L_{9} L_{5} L_{10}^{2}+X L_{9} L_{5} L_{11}^{2}-X L_{9}^{2} L_{6} L_{10}-\right. \\
& \left.X L_{9}^{2} L_{7} L_{11}+Y L_{10}^{3} L_{5}+Y L_{10} L_{5} L_{11}^{2}-Y L_{10}^{2} L_{9} L_{6}\right) ;
\end{aligned}
$$

$b=\left(-Y L_{10} L_{9} L_{7} L_{11}+Z L_{11} L_{5} L_{10}^{2}+Z L_{11}^{3} L_{5}-Z L_{11} L_{9} L_{6} L_{10}-\right.$ $\left.Z L_{11}^{2} L_{9} L_{7}-L_{9} L_{6} L_{10}-L_{9} L_{7} L_{11}\right)$;

$$
\begin{aligned}
& \xi=x-x_{o} ; \\
& \eta=y-y_{o},
\end{aligned}
$$

$x_{o}, y_{o}$ image coordinates of the principal point

$$
\begin{gathered}
r^{2}=\xi^{2}+\eta^{2} ; \\
F(k)=L_{12} r^{2}+L_{13} r^{4}+L_{14} r^{6}
\end{gathered}
$$

= function of symmetrical radial lens distortion;

$\Delta x(p), \Delta y(p)$ are the decentering lens distortion corrections for $x$ and $y$ photo coordinates, and can be obtained by:

$$
\left.\begin{array}{l}
\Delta x(p)=L_{15}\left(r^{2}+2 \xi^{2}\right)+L_{16} \xi \eta \\
\Delta y(p)=L_{15} \eta \xi+L_{16}\left(r^{2}+2 \eta^{2}\right)
\end{array}\right\},
$$

where $L_{2}, \ldots, L_{16}$ are the transformation coefficients.

The linearized form of MDLT Equations with suggesting additional constraints to consider supplemental observation equations, for least squares method solution, can be given by Equation (4) where:

$\Delta$ is the correction vector to the current values set for the unknowns (the 15 MDLT parameters for each photo and object space coordinates of the new points) in the iterative solution;

$B$ is the matrix of the partial derivatives of Equation (9) with respect to the unknowns;

$V$ is the residual vector, i.e., the correction vector to the observations;

$\varepsilon$ is the discrepancy vector;

$\Delta^{c}$ is the vector of observational corrections to the object space coordinates of the control points;

$\varepsilon^{c}$ is the discrepancy vector, between observed values and current (in iterative solution) values of the object space coordinates of the control points.

For starting the iterative solution, approximate values of unknowns should be known. These unknowns are the object space coordinates of new points and the MDLT parameters for each photo.

The method for estimating the approximate values is explained in details in (El-Ashmawy 2006).

\section{Developing and testing the necessary softwares}

The current research includes the development of three softwares for the determination of camera exterior orientations parameters. The softwares are written in Visual $C^{++}$(Gregory 1998) and utilising efficient techniques of Data Structuring, Random File Access and 
Dynamic Memory Allocations for automatic processing and representation of the data and results (Malik 2010). The softwares have been designed to make use of efficient user interfaces (window-driven) for facilitating its execution to the user.

Analytical determination of camera exterior orientation parameters involves extensive computations and the various steps of which are subjected to computational system error. System error consists of two parts (El-Ashmawy 1999). The first part of this error is due to rounding off of values during intermediate computations. This part of error may be minimised by using double precision computations as far as possible. The second part of the system error occurs due to truncation of higher order terms while forming the linearized observation equations from the non linear condition equations.

Mathematical photogrammetric data can be advantageously used for testing of photogrammetric methodologies and systems since in this case error free input data and end results are both known. Testing the developed softwares, therefore, were carried out by using the mathematically generated blocks of photographs of MATHP software (El-Ashmawy 1999).

Out of the various mathematical photogrammetric blocks generated, the block having the shown specifications in Table 1 was used for testing the system error of the developed softwares.

Table 1. Specifications for the generated mathematical photogrammetric block

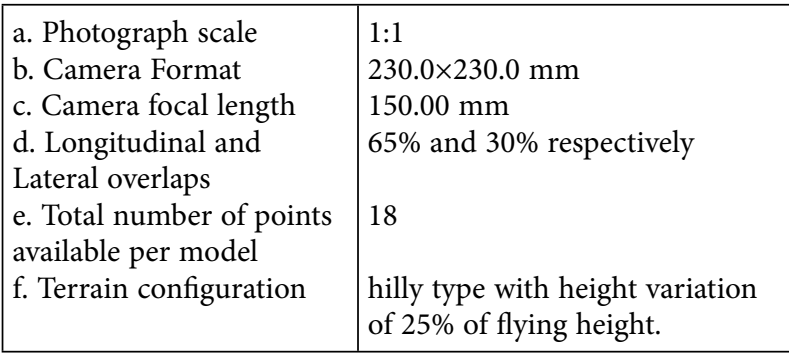

To reduce the effect of the number and location of the control points during the testing phase of the system error, suitable distribution and number of control points have been adopted (El-Ashmawy 1999). The block size was 5 strips each of six photographs. In this case, the block contained 66 and 132 control and check points respectively.

In order to ascertain the accuracy of the results, the root mean square error (RMSE) was computed using the well known formulation:

$R M S E=\sqrt{\sum_{i=1}^{n}(\text { known value }- \text { computed value })_{i}^{2} / n}$.
For each software, the RMSE values for camera exterior orientation parameters and ground coordinates of check and ground control points for this testing phase have been obtained. The results showed that the maximum system error is $0.0002 \mu \mathrm{m}$, at photo scale $1: 1$, for the ground coordinates of check points which is negligible. Therefore, it can be concluded that the developed softwares are free from system error and that they are functional.

\section{Investigating the accuracies of the derived methods using mathematical photogrammetric data}

In this case, error free photogrammetric data of blocks of different sizes using MATHP software were generated as shown in Table 2, and random and lens distortion errors were generated and applied to the error free photo coordinates and ground coordinates of control points of the generated blocks as explained in (El-Ashmawy, Azmi 2003).

Table 2. The configurations for mathematical photogrammetric blocks

\begin{tabular}{|c|c|c|c|c|c|c|}
\hline \multirow[b]{2}{*}{$\begin{array}{c}\text { Block } \\
\text { Title }\end{array}$} & \multicolumn{3}{|c|}{ Block Size } & \multirow[b]{2}{*}{$\begin{array}{l}\text { No. of } \\
\text { Image } \\
\text { Points }\end{array}$} & \multicolumn{2}{|c|}{ Ground Points } \\
\hline & $\begin{array}{l}\text { No. of } \\
\text { Strips }\end{array}$ & $\begin{array}{c}\text { No. of } \\
\text { Photos/ } \\
\text { Strip }\end{array}$ & $\begin{array}{c}\text { No. of } \\
\text { Pho- } \\
\text { tos/ } \\
\text { block }\end{array}$ & & $\begin{array}{l}\text { Cont- } \\
\text { rol } \\
\text { Points }\end{array}$ & $\begin{array}{l}\text { Check } \\
\text { Points }\end{array}$ \\
\hline $\begin{array}{c}1 \\
\text { Model }\end{array}$ & 1 & 2 & 2 & 36 & 6 & 12 \\
\hline 1 Strip & 1 & 5 & 5 & 117 & 15 & 30 \\
\hline $\begin{array}{c}2 \\
\text { Strips }\end{array}$ & 2 & 5 & 10 & 234 & 25 & 50 \\
\hline $\begin{array}{c}3 \\
\text { Strips }\end{array}$ & 3 & 5 & 15 & 351 & 35 & 70 \\
\hline $\begin{array}{c}4 \\
\text { Strips }\end{array}$ & 4 & 5 & 20 & 468 & 45 & 90 \\
\hline $\begin{array}{c}5 \\
\text { Strips }\end{array}$ & 5 & 5 & 25 & 585 & 55 & 110 \\
\hline
\end{tabular}

The random errors were generated, using special error generator (El-Ashmawy, Azmi 2003), within $\pm 11 \mu \mathrm{m}$ and $\pm 8 \mu \mathrm{m}$ for photo coordinates and ground coordinates of control points at photo scale 1:1 respectively. Lens distortion errors were generated by assigning values for the lens distortion coefficients and generating errors in the range of $50 \mu \mathrm{m}$.

Finally, simultaneous adjustment using the derived methods was performed to adjust the available blocks for the determination of camera exterior orientation parameters of each photograph. Then, the 
ground coordinates of points were computed by means of space intersection as explained earlier. The results, in the form of RMSE values at the ground coordinates of points, were obtained and tabulated in Table 3 .

From Table 3 the following conclusions can be drawn:

- The derived mathematical models are suitable for the determination of camera exterior orientation parameters for a photogrammetric block of any size.

- The results of camera exterior orientation parameters using the coplanarity equations are slightly better than the results for using collinearity or DLT method.

- The results of using collinearity or coplanarity condition are slightly better than the results of using DLT method.

\section{Investigating the accuracies of the derived methods using actual photogrammetric data}

A pair of stereo B/W diapositives of Canton de Vaud, Switzerland (El-Ashmawy 1999) was used to investigate the accuracy of the derived methods for the determination of camera exterior orientation parameters. The height difference in the area is about $78.00 \mathrm{~m}$ and mean terrain altitude is $620.00 \mathrm{~m}$ above the mean sea level. The aerial photographs were taken by Wild Avioplot RC10 Automatic Camera System of Echallens of wide angle coverage on a $23 \times 23 \mathrm{~cm}$ format at $1280.00 \mathrm{~m}$ height with focal length $153.18 \mathrm{~mm}$ lens, as a result, the average photo scale is about 1:4300. The camera calibration data such as calibrated focal lens, calibrated fiducial marks and radial lens distortion are available. The area contains 16 well-distributed and identified control points. The control point numbers, ground coordinates and standard errors are also available.

Conventional method of the coordinates measurement of image points was carried out (El-Ashmawy 1999) on the stereo comparator of Aviolyt BC2, Leica, Switzerland, having a least count of $1 \mu \mathrm{m}$.

Seven patterns of control points (El-Ashmawy 1999), where the number of control points ranged from three to twelve points, were chosen in order to compare the photogrammetrically extracted data coordinates with the corresponding ground survey values at check points.

The values of RMSE at the ground coordinates of all points for each control pattern and derived method were obtained as shown in Table 4.

Table 3. The accuracies of the derived methods using mathematical photogrammetric data

\begin{tabular}{|c|c|c|c|c|}
\hline \multirow{2}{*}{ Method } & \multirow{2}{*}{ Block Title } & \multicolumn{3}{|c|}{ RMSE Values for Ground Coordinates $(\mu \mathrm{m})$ at Photo Scale 1:1 } \\
\hline & & $X$ & $Y$ & $Z$ \\
\hline \multirow{6}{*}{ Collinearity } & 1 Model & 5.870 & 8.260 & 12.950 \\
\hline & 1 Strip & 6.24 & 7.740 & 12.390 \\
\hline & 2 Strips & 4.510 & 5.800 & 11.840 \\
\hline & 3 Strips & 3.860 & 4.420 & 8.150 \\
\hline & 4 Strips & 4.290 & 3.890 & 8.280 \\
\hline & 5 Strips & 3.890 & 4.470 & 7.700 \\
\hline \multirow{6}{*}{ Coplanarity } & 1 Model & 5.464 & 7.781 & 12.599 \\
\hline & 1 Strip & 5.808 & 7.311 & 12.014 \\
\hline & 2 Strips & 4.193 & 5.464 & 11.485 \\
\hline & 3 Strips & 3.593 & 4.164 & 7.955 \\
\hline & 4 Strips & 3.991 & 3.662 & 8.042 \\
\hline & 5 Strips & 3.539 & 4.215 & 7.486 \\
\hline \multirow{6}{*}{$\begin{array}{c}\text { Direct Linear } \\
\text { Transformation (DLT) }\end{array}$} & 1 Model & 6.407 & 8.769 & 14.317 \\
\hline & 1 Strip & 6.811 & 8.211 & 13.969 \\
\hline & 2 Strips & 4.946 & 6.152 & 12.229 \\
\hline & 3 Strips & 4.249 & 4.699 & 9.639 \\
\hline & 4 Strips & 4.619 & 4.137 & 9.797 \\
\hline & 5 Strips & 4.213 & 4.754 & 9.119 \\
\hline
\end{tabular}


From Table 4, it can be concluded that:

- The derived mathematical models are suitable for the determination of camera exterior orientation parameters for a block of photographs of any size.

- Increasing the number of control points improves the obtained accuracies.

- Using more than three control points significantly reduces the RMSE values especially for $\mathrm{Z}$ coordinates.

- Using different control distribution patterns, for the same number of control points has an effect on the obtained RMSE values. This is evident from the comparison of RMSE values of $6 \mathrm{~A}$ and $6 \mathrm{~B}$ control distribution patterns. Furthermore, using $6 \mathrm{~B}$ distribution pattern gives smaller RMSE values for planimetry and height than $6 \mathrm{~A}$ distribution pattern.

The results of using the coplanarity equations are slightly better than the results for using collinearity or DLT equations.
The results of using collinearity or coplanarity condition are slightly better than the results of using DLT method.

\section{Conclusions}

Camera exterior orientation parameters for a block of photographs of any size can be determined using the derived mathematical models based on collinearity condition, coplanarity condition and DLT method.

The coplanarity equations have significant effect on compensating the lens distortion errors rather than collinearity equations or DLT method. For this reason, the accuracy of the results of using coplanarity equations is slightly better than using collinearity equations or DLT method.

Compared to the collinearity equations and DLT method, the coplanarity equations has very complex differential coefficients and difficult to be programmed.

Although the results of the DLT method are less accurate than those of using collinearity or coplanarity

Table 4. The accuracies of the derived methods using actual photogrammetric data

\begin{tabular}{|c|c|c|c|c|c|c|}
\hline \multirow{2}{*}{ Method } & \multirow{2}{*}{ Control Pattern } & \multirow{2}{*}{$\begin{array}{l}\text { No. of Control } \\
\text { Points }\end{array}$} & \multirow{2}{*}{$\begin{array}{c}\text { No. of Check } \\
\text { Points }\end{array}$} & \multicolumn{3}{|c|}{ RMSE Values for Ground Coordinates $(\mathrm{cm})$} \\
\hline & & & & $X$ & Y & $Z$ \\
\hline \multirow{7}{*}{ Collinearity } & 3 & 3 & 13 & 5.540 & 6.434 & 14.082 \\
\hline & 4 & 4 & 12 & 6.343 & 5.368 & 10.080 \\
\hline & 5 & 5 & 11 & 5.600 & 5.287 & 9.549 \\
\hline & $6 \mathrm{~A}$ & 6 & 10 & 5.813 & 5.585 & 9.473 \\
\hline & $6 \mathrm{~B}$ & 6 & 10 & 5.731 & 5.310 & 9.439 \\
\hline & 9 & 9 & 7 & 5.351 & 5.468 & 9.335 \\
\hline & 12 & 12 & 4 & 5.005 & 5.714 & 8.998 \\
\hline \multirow{7}{*}{ Coplanarity } & 3 & 3 & 13 & 5.318 & 5.979 & 13.589 \\
\hline & 4 & 4 & 12 & 6.186 & 4.991 & 9.727 \\
\hline & 5 & 5 & 11 & 5.476 & 4.917 & 9.215 \\
\hline & $6 \mathrm{~A}$ & 6 & 10 & 5.584 & 5.411 & 9.122 \\
\hline & $6 \mathrm{~B}$ & 6 & 10 & 5.395 & 5.297 & 9.092 \\
\hline & 9 & 9 & 7 & 5.137 & 5.115 & 9.008 \\
\hline & 12 & 12 & 4 & 4.814 & 5.314 & 8.584 \\
\hline \multirow{7}{*}{$\begin{array}{c}\text { Direct Linear } \\
\text { Transformation } \\
\text { (DLT) }\end{array}$} & 3 & 3 & 13 & 6.160 & 7.003 & 15.347 \\
\hline & 4 & 4 & 12 & 7.048 & 6.175 & 10.953 \\
\hline & 5 & 5 & 11 & 6.153 & 5.975 & 10.379 \\
\hline & $6 \mathrm{~A}$ & 6 & 10 & 6.457 & 6.353 & 10.253 \\
\hline & $6 \mathrm{~B}$ & 6 & 10 & 6.264 & 6.257 & 10.232 \\
\hline & 9 & 9 & 7 & 5.746 & 6.076 & 10.135 \\
\hline & 12 & 12 & 4 & 5.726 & 6.630 & 9.528 \\
\hline
\end{tabular}


condition, the DLT method does not need the known interior orientation nor the exterior orientation parameters. Therefore the DLT method can be used when the necessary information for the collinearity or coplanarity model is not available.

This paper shows the necessity for the mathematical photogrammetric data for testing the photogrammetric methods and softwares.

\section{References}

Abdel-Aziz, Y. I.; Karara, H. M. 1971. Direct linear transformation into object space coordinates in close-range photogrammetry, in Proc. Symposium on Close Range Photogrammetry, June 15-20, 1971, Urbana, Illinois: 1-18.

El-Ashmawy, K. 2006. Feasibility of direct linear transformation (DLT) method for aerial photogrammetry applications, Engineering Research Journal, Shoubra Faculty of Engineering 7: 96-112.

El-Ashmawy, K.; Azmi, M. 2003. Photogrammetric simultaneous and self calibration block adjustments using coplanarity condition, Engineering Research Journal, Faculty of Engineering 87: 85-101.

El-Ashmawy, K. 1999. A Cost-Effective Photogrammetric System for Engineering Applications. Ph.D. Thesis. Roorkee: University of Roorkee.

Elnima, E. E. 2015. A solution for exterior and relative orientation in photogrammetry, a genetic evolution approach, Journal of King Saud University - Engineering Sciences http://dx.doi.org/10.1016/j.jksues.2013.05.004

Ghosh, S. K. 2005. Fundamentals of Computational Photogrammetry. New Delhi: Concept Publishing Company.
Gregory, K. 1998. Special Edition Using Visual C++ 6. USA: Que.

Grussenmeyer, P.; Al Khalil, O. 2002. Solutions for exterior orientation in photogrammetry: a review. The Photogrammetric Record 17: 615-634.

http://dx.doi.org/10.1111/0031-868X.00210.

Li, C.; Zhao, Y. 2012. Approach of camera relative pose estimation based on epipolar geometry, Information Technology Journal 11: 1202-1210.

Malik, D.S. 2010. Data Structures Using C++. $2^{\text {nd }}$ ed. USA: Cengage Learing, Inc.

Pannao, W.; Pintavirooj, C. 2012. Application of direct linear transform for calibration of miniature computed tomography, in Biomedical Engineering International Conference (BMEiCON), IEEE Dec. 5-7, 2012. Ubon Ratchathani: 1-5.

Wolf, P. R.; Dewitt, B. A.; Wilkinson, B. E. 2014. Elements of Photogrammetry with Applications in GIS. $4^{\text {th }}$ ed. USA: McGraw-Hill Education.

Khalid L. A. EL-ASHMAWY (Dr) is associate professor of Surveying \& Digital Mapping at the Department of Civil Engineering, Al-Matria Faculty of Engineering, Helwan University, Egypt. Presently, at Department of Civil Engineering, College of Engineering and Islamic Architecture, Umm Al-Qura University, Makkah, Saudi Arabia.

His area of expertise includes photogrammetry, land surveying, space photography, digital mapping, GIS and development of surveying softwares.

Dr Khalid El-Ashmawy developed many surveying softwares which were published in national and international journals and conferences, such as SoftPhotoMap, PHOTOMAP, MATHP, ScanCal, LandSurMap, Calendar, RSA (Road Safety Assessment), and MathDTM softwares. 